\title{
COMPARAÇÃO ENTRE AS TÉCNICAS DE SHOULDICE MODIFICADA POR BERLINER E A DE FALCI-LICHTENSTEIN NA HERNIOPLASTIA INGUINAL
}

\author{
COMPARISON BETWEEN THE SHOULDICE MODIFIED BY BERLINER AND THE \\ FALCI-LICHTENSTEIN TECHNIQUES IN THE INGUINAL HERNIA REPAIR
}

\author{
Rachid Guimarães Nagem, TCBC- MG; Alcino Lázaro da Silva, ECBC, MG²
}

\begin{abstract}
RESUMO: Objetivo: Comparar os resultados da hernioplastia inguinal pelas técnicas de Shouldice modificada por Berliner (SB), que realiza dois planos de sutura superpostos e de Falci-Lichtenstein (FL) que utiliza prótese de polipropileno. Método: Foram estudados prospectivamente 312 pacientes operados pelo mesmo cirurgião entre 1997 e 2004 . O Grupo 1 constou de 84 pacientes operados pela técnica de FL e o Grupo 2 foi formado por 228 pacientes operados pela técnica de SB. Todos eram masculinos, maiores de 18 anos e portadores de hérnias inguinais tipo 3A, 3B e 4 da classificação de Nyhus. Os grupos foram semelhantes em relação a idade, lado operado, tipo de hérnia, anestesia empregada e tempo de seguimento (média de 3,5 anos). Resultados: A duração média dos procedimentos foi de 53 min no Grupo 1 e de 57 min no Grupo 2 (p=0,2982); a permanência hospitalar foi menor que $24 \mathrm{~h}$ para $94 \%$ dos pacientes do Grupo 1 e $92 \%$ do Grupo 2 ( $\mathrm{p}=0,8050$ ); a incidência de complicações foi de $9,5 \%$ no Grupo 1 e de $12,3 \%$ no Grupo $2(\mathrm{p}=0,5557)$ sendo a mais comum o hematoma/equimose e a taxa de recidiva foi de $1,2 \%$ no Grupo 1 e de $5,4 \%$ no Grupo 2 $(\mathrm{p}=0,0935)$. Nenhum desses resultados apresentou diferença significante. Conclusão: A técnica SB mostrou-se comparável à técnica de FL em homens, maiores de 18 anos com hérnias tipo 3 A, 3 B e 4 de Nyhus, além de não exigir material protético (Rev. Col. Bras. Cir. 2007; 34(5): 331-335).
\end{abstract}

Descritores: Hérnia inguinal; Anestesia local; Canal inguinal; Procedimentos cirúrgicos operatórios; Estudo comparativo.

\section{INTRODUÇÃO}

A hérnia inguinal (HI) é afecção muito prevalente e, consequentemente, seu tratamento é de grande impacto socioeconômico. Inúmeras técnicas de hernioplastia foram e continuam sendo criadas demonstrando não existir procedimento ideal ${ }^{1}$. Atualmente as técnicas de Shouldice e de FL disputam a primazia no reparo por via aberta da HI. A técnica de Shouldice, desenvolvida no Canadá por Earle Shouldice (1945), consiste na abertura da fáscia transversal e confecção de quatro planos de sutura superpostos ${ }^{2}$. Em 1983 Stanley D. Berliner, da State University of New York, mostrou em estudo prospectivo e randomizado, que a realização somente dos dois planos iniciais do reparo à Shouldice apresentava os mesmos resultados quando comparado aos quatro planos originais ${ }^{3}$. A técnica de Falci-Lichtenstein, iniciada com o trabalho de Felício Falci ${ }^{4}$ no Brasil (1969) e posteriormente por Irving Lichtenstein ${ }^{5}$ nos Estados Unidos (1989), preconiza a colocação sistemática de prótese de polipropileno na região inguinal. O objetivo deste trabalho foi comparar a técnica de Shouldice modificada por Berliner com a técnica de Falci-Lichtenstein.

\section{MÉTODO}

Foram estudados, prospectivamente, 312 pacientes consecutivos operados em dois hospitais da região metropo- litana de Belo Horizonte entre julho de 1997 e agosto de 2004 pelo mesmo cirurgião. Cada um daqueles hospitais possuía seu próprio protocolo para o tratamento da HI. Enquanto um deles tratava pacientes masculinos, maiores de 18 anos, com hérnias inguinais tipo 3 A, 3 B e 4 da classificação de Nyhus pela técnica de FL., o outro tratava pacientes com o mesmo tipo de hérnia pela técnica de SB.

O Grupo 1 foi composto de 84 pacientes portadores de 91 hérnias inguinais (sete bilaterais) operados pela técnica de FL. O Grupo 2 foi formado por 228 pacientes portadores de 241 hérnias inguinais (13 bilaterais) operados pela técnica de SB (Figuras 1 e 2).

Para avaliar sua comparabilidade, os dois grupos foram analisados quanto às características da casuística: idade, lado acometido pela hérnia, tipo de hérnia pela classificação de Nyhus, tipo de anestesia empregado e período de seguimento pós-operatório.

Para análise das recidivas relacionou-se 266 pacientes ( $85,25 \%$ da casuística inicial). Essa diminuição deveu-se a algumas perdas de acompanhamento e, também, pela exclusão dos pacientes com seguimento inferior à dois anos (para dar maior consistência aos dados). No Grupo 1, 75 pacientes com 82 reparos (sete bilaterais) tiveram acompanhamento de dois anos ou mais enquanto no Grupo 2 foram 191 pacientes com 202 reparos (11 bilaterais). Calculou-se as taxas de recidiva geral, nas hérnias primárias e nas hérnias recidivadas.

1. Cirurgião Geral do Hospital dos Servidores do Estado de Minas Gerais (IPSEMG).

2. Professor Emérito da Faculdade de Medicina da Universidade Federal de Minas Gerais.

Recebido em 02/03/2007

Aceito para publicação em 03/05/2007

Conflito de interesses: nenhum

Fonte de financiamento: nenhuma

Trabalho realizado no Hospital dos Servidores do Estado de Minas Gerais em Belo Horizonte e Hospital Regional Prof. Osvaldo Franco em Betim, M.G. 


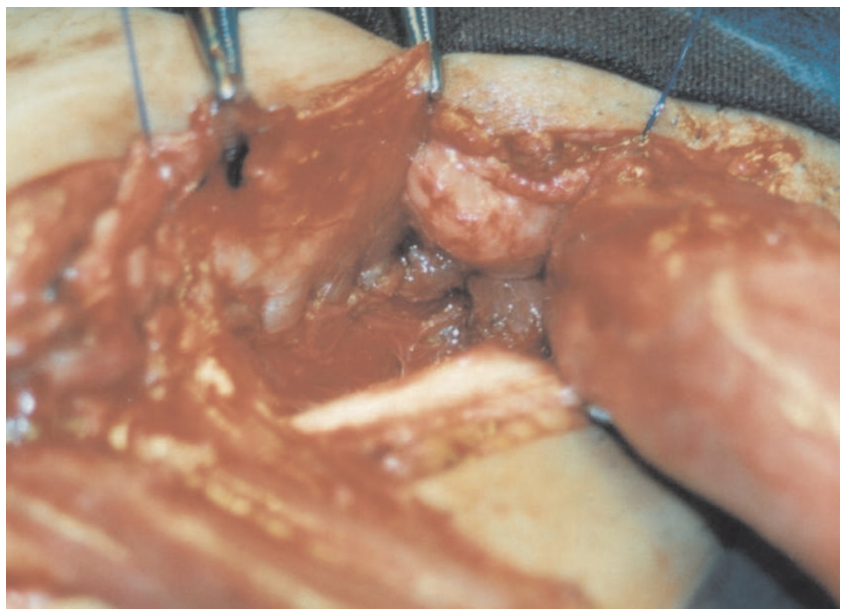

Figura 1 - Término do primeiro plano do reparo à $S B$.

Para análise das características da casuística e dos resultados foram utilizados os testes do qui-quadrado e de Mann-Whitney. Em amostras pequenas (frequência menor que cinco) foi usado o teste exato de Fisher. O nível de significância utilizado foi de $5 \%(\mathrm{p}=0,05)$.

Trabalho aprovado pelo Comitê de Ética em Pesquisa do Instituto de Previdência dos Servidores do Estado de Minas Gerais-IPSEMG (Parecer 193/06)

\section{RESULTADOS}

As características da casuística foram semelhantes nos dois grupos estudados. A média de idade no Grupo 1 foi de 57 anos (18 a 84 anos) e no Grupo 2 de 52 anos (18 a 87 anos). No Grupo 1 constatou-se 50 hérnias inguinais à direita, 27 à esquerda e sete bilaterais, enquanto no Grupo 2 foram 127 hérnias no lado direito, 88 à esquerda e 13 bilaterais. Quanto ao tipo de hérnia pela classificação de Nyhus, o Grupo 1 apresentou 51 hérnias do tipo $3 \mathrm{~B}, 30$ do tipo $3 \mathrm{~A}$ e 10 do tipo 4 enquanto no Grupo 2 foram 123 hérnias tipo 3 B, 92 tipo 3 A e 26 tipo 4. Em relação à anestesia, no Grupo 1 houve 69 raquianestesias, nove peridurais, cinco locais e uma geral enquanto no Grupo 2 foram 184 raquianestesias, 33 peridurais, nove locais e duas gerais. $\mathrm{O}$ tempo de seguimento médio foi de 3,35 anos (dois a sete anos) no Grupo 1 e de 3,64 anos (dois a oito anos) no Grupo 2. Nenhuma dessas variáveis mostrou diferença significante sendo os dois grupos considerados, portanto, comparáveis.

A duração dos procedimentos no Grupo 1 variou de $35 \mathrm{~min}$ a 90min com média de $54 \mathrm{~min}$ e no Grupo 2 ficou entre $30 \mathrm{~min}$ e $120 \mathrm{~min}$ com média de $58 \mathrm{~min}$ ( $\mathrm{p}=0,2982$ ). No Grupo 1 a alta hospitalar com menos de $24 \mathrm{~h}$ ocorreu em $94,1 \%$ dos pacientes e no Grupo 2 em 92,6\% ( $\mathrm{p}=0,8050$ ). O Grupo 1 apresentou 9,5\% de complicações enquanto no Grupo 2 foram $12.3 \%$ ( $\mathrm{p}=0,5557)$. As complicações mais frequentes estão relacionadas na Tabela 1.

Houve uma recidiva no Grupo 1 (1,2\%) num paciente com 73 anos com hérnia bilateral recidivada à esquerda, passado de prostatectomia radical e incontinência urinária. Foi feito o reparo bilateral, iniciando-se pelo lado esquerdo, e ocorreu recidiva à esquerda após seis meses.

No Grupo 2 ocorreram 11 recidivas $(5,4 \%)$ sendo que seis pacientes foram novamente operados no próprio Serviço.

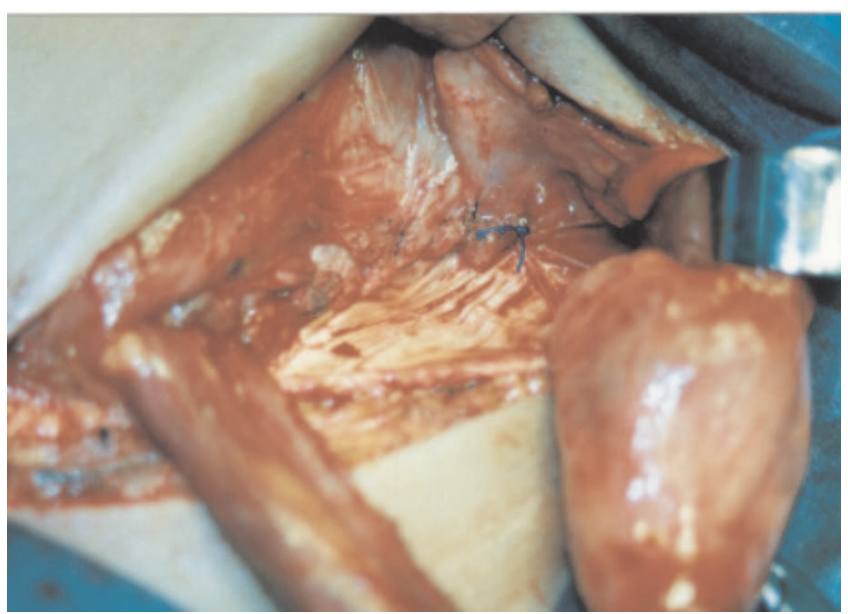

Figura 2 - Término do segundo e último plano do reparo à $S B$.

Quatro desses apresentavam recidiva na forma de hérnia indireta (Figura 3) e dois na forma femoral (Figura 4). Em todos eles a parede posterior onde foi realizado o reparo à SB estava íntegra. A Tabela 2 mostra as características dos pacientes que recidivaram no Grupo 2.

A taxa geral de recidivas foi de $1,2 \%$ no Grupo 1 e de $5,4 \%$ no Grupo 2. Se considerarmos apenas as hérnias primárias a taxa de recidiva foi de $0 \%$ no Grupo 1 e de 4,4\% (8 em 178) no Grupo 2 ( $\mathrm{p}=0,0630)$. Já com relação às hérnias recidivadas essa taxa foi de $10 \%$ (1 em 10) no Grupo 1 e de $12,5 \%$ (3 em 24) no Grupo 2 ( $p=0,6655)$. Nenhum desses valores mostrou diferença significante.

\section{DISCUSSÃO}

A técnica de FL é hoje considerada o padrão-ouro para o reparo da HI pelo American College of Surgeons ${ }^{6}$. Dessa forma é em relação à ela que novas técnicas devem ser comparadas. A técnica de Shouldice é bastante conhecida mas não se

Tabela 1 - Comparação por complicações específicas em pacientes submetidos à hernioplastia inguinal pelas técnicas de Falci-Lichtenstein (Grupo 1, $n=84$ ) e de Shouldice modificada por Berliner (Grupo 2, $n=228$ ).

\begin{tabular}{llll}
\hline $\begin{array}{l}\text { Principais } \\
\text { complicações }\end{array}$ & $\mathbf{1}$ & Grupo & Valor $\mathbf{p}^{*}$ \\
\hline Dor & 2 & 1 & \\
Hematoma & $2,4 \%$ & $0,5 \%$ & 0,1597 \\
& 3 & 19 & 0,1339 \\
Hidrocele & $3,3 \%$ & $7,9 \%$ & \\
ISC & 0 & 1 & 0,7267 \\
& $0 \%$ & $0,4 \%$ & \\
Orquite & 3 & 9 & 0,5733 \\
& $3,3 \%$ & $3,7 \%$ & \\
Total & 1 & 1 & 0,7259 \\
\hline
\end{tabular}

* Teste exato de Fisher.

ISC: infecção de sítio cirúrgico. 


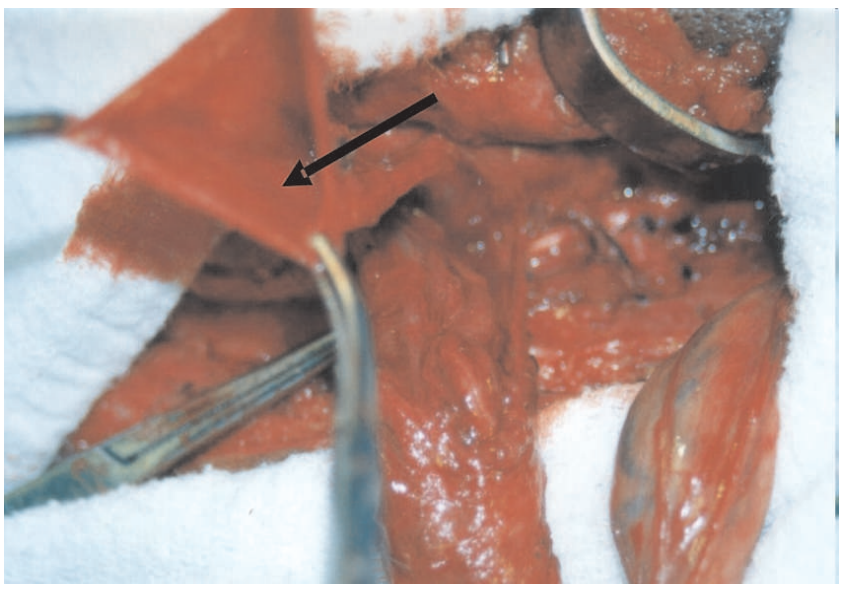

Figura 3 - Recidiva indireta (seta maior). Observar reparo prévio na parede posterior íntegro (seta menor).

encontrou difusão na literatura da modificação feita por Berliner e isso estimulou a realização deste trabalho. Não houve randomização. Cada hospital, já antes de se imaginar esse estudo, tratava seus pacientes masculinos maiores de 18 anos com hérnias tipo 3 A, 3B e 4 de Nyhus com técnica distinta (FL ou $\mathrm{SB})$. Foi criado um protocolo e feito acompanhamento dos pacientes operados em cada hospital para posterior comparação.

As características da casuística foram semelhantes nos dois grupos. A média de idade na $6^{\text {a }}$ década de vida foi similar à literatura. A predominância do lado direito também pôde ser constatada na maioria das publicações e se relaciona com o fato do conduto peritoniovaginal direito demorar mais que o esquerdo para se obliterar ${ }^{7}$. O tipo $3 \mathrm{~B}$ de Nyhus foi o mais frequente no estudo e esta predominância das hérnias indiretas é bem descrita nos tratados de herniologia ${ }^{1}$. O bloqueio anestésico foi empregado na maioria dos casos. Apesar de vários auto-

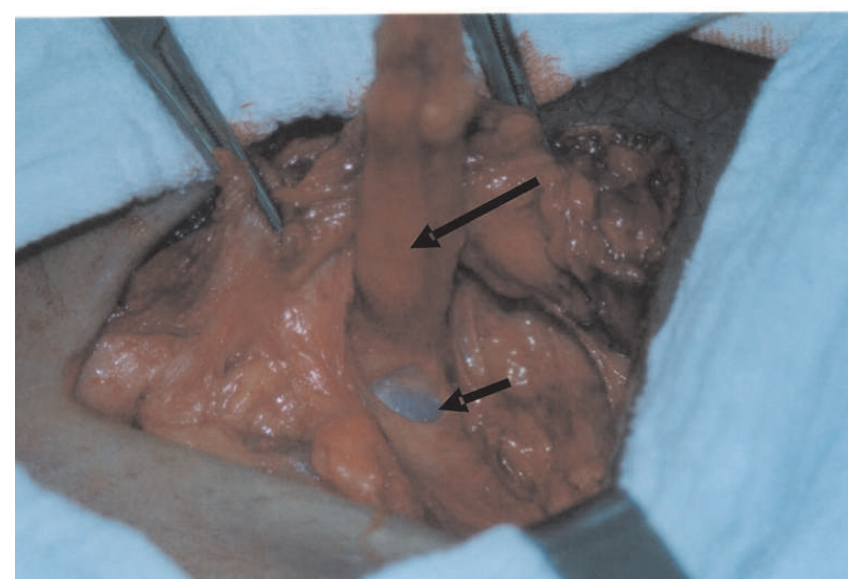

Figura 4 - Recidiva femoral (seta maior). Observar a veia femoral (seta menor).

res também utilizarem preferencialmente o bloqueio anestésico, a tendência é o uso da anestesia local nas hernioplastias inguinais. Tanto as clínicas Lichtenstein e Shouldice quanto Berliner utilizam preferencialmente anestesia local. Seu uso é preferível por ser segura, simples, efetiva e econômica ${ }^{6}$.

A duração dos procedimentos foi similar à da literatura. Apesar da técnica de Shouldice ser mais complexa que a técnica de FL, nesse estudo foi usada uma modificação mais simples dessa técnica, contribuindo para diminuir o tempo operatório.

A permanência hospitalar também não diferiu da maioria das séries. Existe hoje uma clara tendência pela alta hospitalar precoce. Em 1892, quando Bassini publicou seus resultados em 262 pacientes, a média de internação era de 21 dias $^{8}$. Avanços nas áreas de anestesiologia, controle de infecções, técnica operatória, além da questão dos custos contribuíram para diminuição dessa média. Atualmente existem serviços em

Tabela 2 - Características dos pacientes que apresentaram recidiva herniária após hernioplastia inguinal pela técnica de Shouldice modificada por Berliner (grupo 2, $n=228$ ).

\begin{tabular}{lcccccl}
\hline Paciente & Idade(anos) & Comorbidade & Tipo de Hérnia & Tabagismo & $\begin{array}{c}\text { Complicação } \\
\text { pós-operatória }\end{array}$ & Tipo de Recidiva \\
\hline 1 & 75 & HAS & $3 \mathrm{~A}$ & NÃO & NÃO & NÃOREOPERADO \\
2 & 36 & NÃO & $3 \mathrm{~B}$ & SIM & NÃO & INDIRETA \\
3 & 55 & HAS & $3 \mathrm{~A}$ & NÃO & NÃO & INDIRETA \\
4 & 54 & DMOBESIDADE & 4 & SIM & NÃO & NÃOREOPERADO \\
5 & 41 & HIV+ & $3 \mathrm{~B}$ & NÃO & NÃO & INDIRETA \\
6 & 48 & NÃO & $3 \mathrm{~B}$ & NÃO & NÂO & INDIRETA \\
7 & 55 & HIV+ & $3 \mathrm{~B}$ & SIM & NÃO & FEMORAL \\
8 & 64 & HAS & 4 & SIM & NÃO & NÃOREOPERADO \\
9 & 39 & NÃO & $3 \mathrm{~B}$ & SIM & NÃO & FEMORAL \\
10 & 58 & HAS & 4 & NÃO & NÃO & NÃOREOPERADO \\
11 & 79 & HAS Obesidade & $3 \mathrm{~B}$ & NÃO & ISC & NÃOREOPERADO \\
& IRC & & & & \\
\hline
\end{tabular}

HAS-Hipertensão arterial sistêmica.

DM-Diabetes Melito.

HIV +-Portador do vírus da imunodeficiência humana.

IRC-Insuficiência renal crônica.

ISC-Infecção de sítio cirúrgico. 
que os pacientes deixam o hospital duas horas após o término da operação ${ }^{9}$.

A incidência de complicações parece alta à primeira vista mas foi comparável à literatura ${ }^{10}$. Hematomas e equimoses resultam de sangramento originado em qualquer nível anatômico e na maioria das vezes são tratados conservadoramente. Nesse trabalho nenhum paciente necessitou retornar ao bloco cirúrgico. Dois pacientes que apresentaram hematomas procuraram o setor de emergência onde a ferida foi explorada pelo cirurgião plantonista. Ambos evoluíram com Infecção de sítio cirúrgico (ISC). A taxa de ISC também parece alta mas encontra respaldo na literatura. Estudos prospectivos com metodologia rigorosa revelaram taxas de ISC após hernioplastia inguinal entre 3,3\% e $14,04 \%$ com média de 5,8\% ${ }^{11}$. Uma série de 14442 hernioplastias inguinais na Clínica Shouldice revelou formação de hidroceles em $0,7 \%$ dos casos, valor similar ao encontrado neste trabalho. Está associada com excessiva dissecção do funículo espermático e consequente lesão dos ductos linfáticos ${ }^{12}$. Além disso, quando a porção distal do saco herniário que atinge o escroto não é removida, pode haver acúmulo de serosidade no mesmo, sendo considerada hidrocele. Para evitá-las recomenda-se incisar a parede anterior da porção distal do saco herniário indireto, quando não ressecado ${ }^{6}$. Orquite isquêmica foi uma complicação incomum nesse estudo, ela é definida como inflamação pósoperatória do testículo. Sua patogênese envolve isquemia arterial, venosa ou ambas ${ }^{12}$. Possui incidência de $1 \%$ após reparo de hérnias primárias e de $5 \%$ após reparo de hérnias recidivadas, sendo mais comum nas hérnias indiretas inguinoescrotais volumosas. Nesses casos é recomendável a transsecção do saco herniário, deixando-se sua porção distal in situ ${ }^{13}$. Inguinodinia é a dor crônica após reparo da hérnia inguinal. A persistência de dor significativa após um ano do reparo era considerada rara porém publicações recentes relataram até $29 \%$ dessa complicação ${ }^{14}$. Condon questiona uma relação entre esse crescimento e a difusão do uso de próteses ${ }^{15}$. De fato, neste estudo a dor crõnica foi $5 \mathrm{x}$ mais frequente no Grupo 1 em relação ao Grupo 2, porém sem significância. A revisão dos prontuários de 100 pacientes na Clínica Shouldice revelou "preservação cuidadosa" dos nn. ilioinguinal e iliohipogástrico em 90 deles ${ }^{12}$. A própria técnica de Shouldice determina a secção sistemática do ramo genital do nervo genitofemoral ao ressecar-se o m.cremaster, poupando-o nas mulheres onde responde pela sensibilidade dos grandes lábios ${ }^{16}$. Estudo prospectivo duplo-cego randomizou dois grupos, com e sem ressecção do n. ilioinguinal, em pacientes operados pela técnica de Falci-Lichtenstein. O resultado foi menor incidência de dor crônica no grupo submetido à ressecção $(\mathrm{p}=0,008)$ sem que isso tenha aumentado a incidência de dormência ou alterações tróficas na região ${ }^{17}$.

A única recidiva do Grupo 1 ocorreu rente ao pube destacando a importância da prótese ultrapassá-lo em $2 \mathrm{~cm}^{18}$. A análise das recidivas no Grupo 2 revela casos de tabagismo e alguns pacientes idosos, ambos fatores de risco independentes para recidiva ${ }^{19}$. Observa-se também dois portadores do HIV. Existe maior incidência de hérnias da parede abdominal nesses pacientes, porém é incerto se isso ocorre pela infecção ou pelo uso da terapia anti-retroviral ${ }^{20}$. É, sem dúvida, interessante campo para investigação. Todos os pacientes reoperados mostraram a parede posterior, onde foi feito reparo prévio à $\mathrm{SB}$, firme. As recidivas ocorreram no anel interno ou no anel femoral. Recidivas na forma femoral são sabidamente relacionadas à técnica de Shouldice cuja linha de sutura tensionaria o anel femoral, alargando-o ${ }^{21}$. Quanto às recidivas na forma de hérnias indiretas, a Clínica Shouldice enfatiza não poderem existir. Segundo aqueles autores elas representam sacos herniários indiretos que passaram despercebidos na primeira intervenção ${ }^{21,22}$. Para que tal não ocorra recomendam dissecção meticulosa do anel inguinal interno e a busca da cicatriz peritoneal na face medial do anel junto ao ducto deferente, sua identificação descarta a presença de hérnia indireta $^{16}$. A taxa de recidivas foi comparável com as duas técnicas. Em relação às clínicas Shouldice, Lichtenstein e outras esses valores foram elevados, porém aqueles são centros especializados no tratamento da HI. Grandes revisões incluindo hospitais gerais e pequenos centros demostraram média geral de recidiva nos reparos primários de $10 \%$ chegando a $25 \%$ nos recidivantes ${ }^{23}$. Estudo prospectivo e randomizado feito em hospitais de veteranos nos E.U.A.com a técnica de FL mostrou recidiva de $4 \%$ nas hérnias primárias e de $14,1 \%$ nas recidivadas ${ }^{24}$. Apenas dois estudos prospectivos randomizados mostraram diferença significante entre a técnica de Shouldice e de FL (não há estudos desse tipo comparando a modificação proposta por Berliner com a técnica de FL). Em um os reparos foram feitos por médicos residentes ${ }^{25} \mathrm{e} o$ outro elegeu $\mathrm{p}=0,10$ como nível de significância ${ }^{26}$. Esses detalhes devem ser considerados quando se interpretam tais estudos.

Concluiu-se que a técnica de SB apresenta resultados comparáveis aos da técnica de FL com relação à duração do procedimento, permanência hospitalar, complicações e recidiva herniária em homens maiores de 18 anos com hérnias do tipo 3 A, 3 B e 4 da classificação de Nyhus.

\begin{abstract}
Background: To compare the results of the inguinal hernia repair with the Falci-Lichtenstein $(F L)$ tecnique that uses polypropylene mesh and the Shouldice modified by Berliner (SB) tecnique, a repair in two layers of continuous suture. Methods: It is presented a prospective study of 312 patients operated on between 1997 and 2004 by one single surgeon. Group 1 was composed for 84 patients treated with the FL repair and group 2 for 228 patients with the SB repair. All the patients were men, over 18 years, with types $3 \mathrm{~A}, 3 \mathrm{~B}$ and 4 hernias according to the Nyhus classification. The groups were similar in age, operated side, hernia type, anestesia and follow up period. Results: Mean operative time was 53min in group 1 and 57 min in group $2(p=0,2982)$; length of hospital stay was 1 day in $94 \%$ of the cases in group 1 and $92 \%$ in group $2(p=0,8050)$; postoperative complication rate was 9,5\% in group 1 and 12,3\% in group $2(p=0,5557)$ and the most frequent was hematoma/ecchymosis. The recurrence rate at 3,5 years mean follow up time was 1,2\% in group 1 and $5,4 \%$ in group $2(p=0,0935)$. None of these results were significant. Conclusion: The SB repair proved to be as good as the FL tecnique in men, over 18 years with type $3 \mathrm{~A}, 3 \mathrm{~B}$ and 4 inguinal hernias; besides it do not require a mesh prosthesis.
\end{abstract}

Key words: Hernia, inguinal; Anesthesia, local; Inguinal canal; Surgical procedures, operative; Comparative study. 


\section{REFERÊNCIAS}

1. Lázaro da Silva A. Hérnias. $2^{a}$ ed. São Paulo: Roca; 2006.

2. Welsh DR, Alexander MA. The Shouldice repair. Surg Clin North Am. 1993;73(3):451-69.

3. Berliner SD. An approach to groin hernia. Surg Clin North Am. 1984;64(2):197-213.

4. Falci F. Marlex mesh no tratamento da hérnia inguinal do adulto (análise de 100 casos operados). O Hospital. 1969;75(1):14759.

5. Amid PK, Shulman AG, Lichtenstein IL. Open "tension-free" repair of inguinal hernias: the Lichtenstein technique. Eur J Surg. 1996;162(6):447-53

6. Amid PK. Lichtenstein tension-free hernioplasty: its inception, evolution, and principles. Hernia. 2004;8(1):1-7. Epub 2003 Sep 20.

7. Wantz GE. A 65-year-old man with an inguinal hernia. JAMA. 1997;277(8):663-9.

8. Gilbert AI. Day surgery for inguinal hernia. Int Surg. 1995;80(1):48.

9. Kurzer M, Belsham PA, Kark AE. The Lichtenstein repair. Surg Clin North Am. 1998;78(6):1025-46.

10. Porrero JL, Hidalgo M, Sanjuanbenito A, Sanchez-Cabezudo C. The Shouldice herniorrhaphy in the treatment of inguinal hernias: a prospective study on 775 patients. Hernia. 2004;8(1):60-3. Epub 2003 Nov 26.

11. Yerdel MA, Akin EB, Dolalan S, Turkcapar AG, Pehlivan M, Gecim IE, Kuterdem E. Effect of single-dose prophylactic ampicillin and sulbactam on wound infection after tension-free inguinal hernia repair with polypropylene mesh: the randomized, double-blind, prospective trial. Ann Surg. 2001;233(1):26-33.

12. Bendavid R. Complications of groin hernia surgery. Surg Clin North Am. 1998;78(6):1089-103.

13. Wantz GE. Testicular complications of inguinal hernioplasty. Probl Gen Surg. 1995;12:219-24.

14. Bay-Nielsen M, Perkins FM, Kehlet H; Danish hernia Database. Pain and functional impairment 1 year after inguinal herniorrhaphy: a nationwide questionnaire study. Ann Surg. 2001;233(1):1-7.

15. Condon RE. Groin pain after hernia repair. Ann Surg. 2001;233(1):8.

16. Nyhus LM, Baker RJ. Mastery of surgery. $2^{\text {nd }}$ ed. Boston: Little, Brown and Company; 1992.

17. Mui WL, Ng CS, Fung TM, Cheung FK, Wong CM, Ma TH, Bn MY, Ng EK. Prophylactic ilioinguinal neurectomy in open inguinal hernia repair: a double-blind randomized controlled trial. Ann Surg. 2006;244(1):27-33.
18. Amid PK. The Lichtenstein repair in 2002: an overview of causes of recurrence after Lichtenstein tension-free hernioplasty. Hernia. 2003;7(1):13-6. Epub 2002 Oct 5.

19. Junge K, Rosch R, Klinge U, Schwab R, Peiper Ch, Binnebösel $\mathrm{M}$, Schenten F, Schumpelick V. Risk factors related to recurrence in inguinal hernia repair: a retrospective analysis. Hernia. 2006;10(4):309-15. Epub 2006 May 23.

20. Morfeldt L, Sundstrom A, R Olofsson R, Thulin G, Åkerlund B, Koppel K, Karlsson A, Flamholc L, Håkangård C, Granholm H, Liedberg A, Mortimer Ö; The HivBivus Collaborative Group. Hernia of the abdominal wall: a high incidence in HIV-infected patients. Antiviral Ther. 2001;6(Suppl 4):64.

21. Wantz GE. The Canadian repair: personal observations. World J Surg. 1989;13(5):516-21.

22. Lowham AS, Filipi CJ, Fitzgibbons RJ, Stoppa R, Wantz GE, Felix EL, Crafton WB. Mechanisms of hernia recurrence after preperitoneal mesh repair. Traditional and laparoscopic. Ann Surg. 1997;225(4):422-31.

23. Rutkow IM, Robbins AW. Demographic, classificatory, and socioeconomic aspects of hernia repair in the United States. Surg Clin North Am. 1993;73(3):413-26.

24. Neumayer L, Giobbie-Hurder A, Jonasson O, Fitzgibbons R, Dunlop D, Dunlop D, Gibbs J, Reda D, Henderson W; Veterans Affairs Cooperative Studies Program 456 Investigators. Open mesh versus laparoscopic mesh repair of inguinal hernia. $\mathrm{N}$ Engl J Med. 2004;350(18):1819-27. Epub 2004 Apr 25.

25. Danielsson P, Issacson S, Hansen MV. Randomised study of Lichtenstein compared with Shouldice inguinal hernia repair by surgeons in training. Eur J Surg. 1999;165(1):49-53.

26. McGillicuddy JE. Prospective randomized comparison of the Shouldice and Lichtenstein hernia repair procedures. Arch Surg. 1998;133(9):974-8.

Como citar este artigo:

Nagem RG, Silva AL. Comparação entre as técnicas de Shouldice modificada por Berliner e de Falci-Lichtenstein na hernioplastia inguinal. Rev Col Bras Cir. [periódico na Internet] 2007 Set - Out; 34(5). Disponível em URL: www.scielo.br/rcbc

Endereço para correspondência:

Rachid Guimarães Nagem

Rua Gonçalves Dias, 332 - apto. 1001

Bairro Funcionários

30140-090 - Belo Horizonte - MG

Tel: (31) 3214-0141 - (31) 99573710) - (31) 30479850

Fax: (31) 3214-0141

E-mail:rgnagem@yahoo.com.br 